\title{
Mechanisms of protective effects of astaxanthin in nonalcoholic fatty liver disease
}

\author{
Ling-Jia Gao, Yu-Qin Zhu, Liang Xu \\ School of Laboratory Medicine and Life Sciences, Wenzhou Medical University, Wenzhou 325035, Zhejiang, China. \\ Correspondence to: Liang Xu, School of Laboratory Medicine and Life Sciences, Wenzhou Medical University, Chashan \\ University Town, Wenzhou 325035, Zhejiang, China. E-mail: wanzhe1023@126.com
} How to cite this article: Gao LJ, Zhu YQ, Xu L. Mechanisms of protective effects of astaxanthin in nonalcoholic fatty liver disease.
Hepatoma Res 2021;7:30. https://dx.doi.org/10.20517/2394-5079.2020.150

Received: 20 Nov 2020 First Decision: 24 Dec 2020 Revised: 29 Dec 2020 Accepted: 6 Jan 2021 Available online: 9 Apr 2021

Academic Editor: Stefano Bellentani Copy Editor: Miao Zhang Production Editor: Jing Yu

\begin{abstract}
Nonalcoholic fatty liver disease is a major contributor to chronic liver disease worldwide, and 10\%-20\% of nonalcoholic fatty liver progresses to nonalcoholic steatohepatitis (NASH). Astaxanthin is a kind of natural carotenoid, mainly derived from microorganisms and marine organisms. Due to its special chemical structure, astaxanthin has strong antioxidant activity and has become one of the hotspots of marine natural product research. Considering the unique chemical properties of astaxanthin and the complex pathogenic mechanism of NASH, astaxanthin is regarded as a significant drug for the prevention and treatment of NASH. Thus, this review comprehensively describes the mechanisms and the utility of astaxanthin in the prevention and treatment of NASH from seven aspects: antioxidative stress, inhibition of inflammation and promotion of M2 macrophage polarization, improvement in mitochondrial oxidative respiration, regulation of lipid metabolism, amelioration of insulin resistance, suppression of fibrosis, and liver tumor formation. Collectively, the goal of this work is to provide a beneficial reference for the application value and development prospect of astaxanthin in NASH.
\end{abstract}

Keywords: Nonalcoholic fatty liver disease, astaxanthin, fibrosis, insulin resistance, mitochondrial dysfunction, oxidative stress

\section{INTRODUCTION}

Nonalcoholic fatty liver disease (NAFLD) has become one of the most prevalent forms of chronic liver disease in most countries, and is frequently associated with obesity, metabolic syndrome, and type 2 diabetes $^{[1]}$. NAFLD is characterized by the accumulation of triglyceride (TG) fats by more than $5 \%$ to $10 \%$ of the liver weight in the absence of superfluous alcohol consumption. Nonalcoholic steatohepatitis (NASH),

(C) The Author(s) 2021. Open Access This article is licensed under a Creative Commons Attribution 4.0 International License (https://creativecommons.org/licenses/by/4.0/), which permits unrestricted use, sharing, adaptation, distribution and reproduction in any medium or format, for any purpose, even commercially, as long as you give appropriate credit to the original author(s) and the source, provide a link to the Creative Commons license, and indicate if changes were made. 
an advanced form of NAFLD, is characterized by hepatocellular steatosis, lobular inflammation, and fibrosis, and may lead to liver cirrhosis and hepatocellular carcinoma ${ }^{[2-4]}$. According to global epidemiological research, the global prevalence of NAFLD is increasing year by year and reached approximately $25 \%$ by $2016^{[5-7]}$. Studies have shown that NAFLD is rapidly increasing as an indicator for liver transplantation, and its incidence in the United States is currently as high as one-third of the total population $^{[8]}$.

For the pathogenesis of NASH, the widely accepted theory is the "second or multiple hits" hypothesis ${ }^{[9,10]}$, as show as Figure 1. Although insulin resistance, enhanced oxidative stress followed by lipid peroxidation, and rising proinflammatory cytokine release are believed to be the major causes of progression to $\mathrm{NASH}^{[1,12]}$, the concrete mechanisms remains obscure. Currently, NAFLD is considered to be an integral part of metabolic syndrome with insulin resistance as the central risk factor. Metabolic syndrome is often characterized by oxidative stress, a disturbance in the balance between the production of reactive oxygen species (ROS) and antioxidant defenses ${ }^{[13]}$.

Currently, several pharmacological agents, such as metformin, thiazolidinediones, and vitamin E have been tested as treatments for NASH in clinical trials ${ }^{[14-16]}$. However, these agents are generally insufficient to ameliorate liver inflammation and fibrosis, and have raised safety concerns. Therefore, a potential therapy with minimal adverse effects is eagerly awaiting. It has recently become clear that the effects of astaxanthin go beyond its antioxidant properties. Astaxanthin is a xanthophyll carotenoid found in marine organisms, including salmon, shrimp, crustaceans, and algae such as Haematococcus pluvialis ${ }^{[17,18]}$. Accumulating evidence suggests that astaxanthin could prevent or even reverse NASH by improving oxidative stress, inflammation, lipid metabolism, insulin resistance and fibrosis.

The objective of this review is to summarize the bio-functions of astaxanthin in the prevention of NASH.

\section{SOURCE, SYNTHETIC AND BIOLOGICAL ACTIVITY OF ASTAXANTHIN}

Astaxanthin is a secondary carotenoid with a chemical structure of $3,3^{\prime}$-dihydroxy-4,4'-diketo- $\beta, \beta^{\prime}$-carotene, which is known for its strong antioxidant activity. It is widely found in nature, such as leaves, flowers, fruits, feathers of flamingos, most fishes, members of the frog family, crustaceans, and the unicellular alga, Haematococcus pluvialis, which is the most ideal source of natural astaxanthin ${ }^{[17,19]}$. At present, astaxanthin is mainly obtained by the biological extraction of aquatic products and by artificial synthesis from carotene as the raw material. Because synthetic astaxanthin is expensive and less natural than natural astaxanthin in terms of chemical safety and biofunctionality, currently astaxanthin is almost always obtained by biological extraction for its use as a dietary supplement.

At present, astaxanthin is widely used in the food industry as a dietary supplement in a growing number of countries. In 1987, the US Food and Drug Administration (FDA) approved astaxanthin as a feed additive for animal and fish feed; in 1999, the FDA authorized it as a dietary supplement for humans ${ }^{[20]}$. Astaxanthin is lipophilic and hydrophilic, it is absorbed by intestinal epithelial cells in the small intestine and then esterified. It is passively diffused and combined with fat molecules. The unesterified part is combined with chylomicrons and then passed through the lymphatic system for transport into the liver. Spiller and Dewell ${ }^{[21]}$ used a randomized, controlled, double-blind approach to assess the safety of oral administration of astaxanthin $(6 \mathrm{mg} / \mathrm{d})$ in healthy individuals. There was no significant difference in blood pressure and various biochemical parameters at 4 and 8 weeks. Humans cannot synthesize astaxanthin, and the ingested astaxanthin cannot be converted to vitamin A; excessive intake of astaxanthin will thus not cause hypervitaminosis $\mathrm{A}^{[22,23]}$. Astaxanthin has physiological functions such as inhibiting tumorigenesis, 


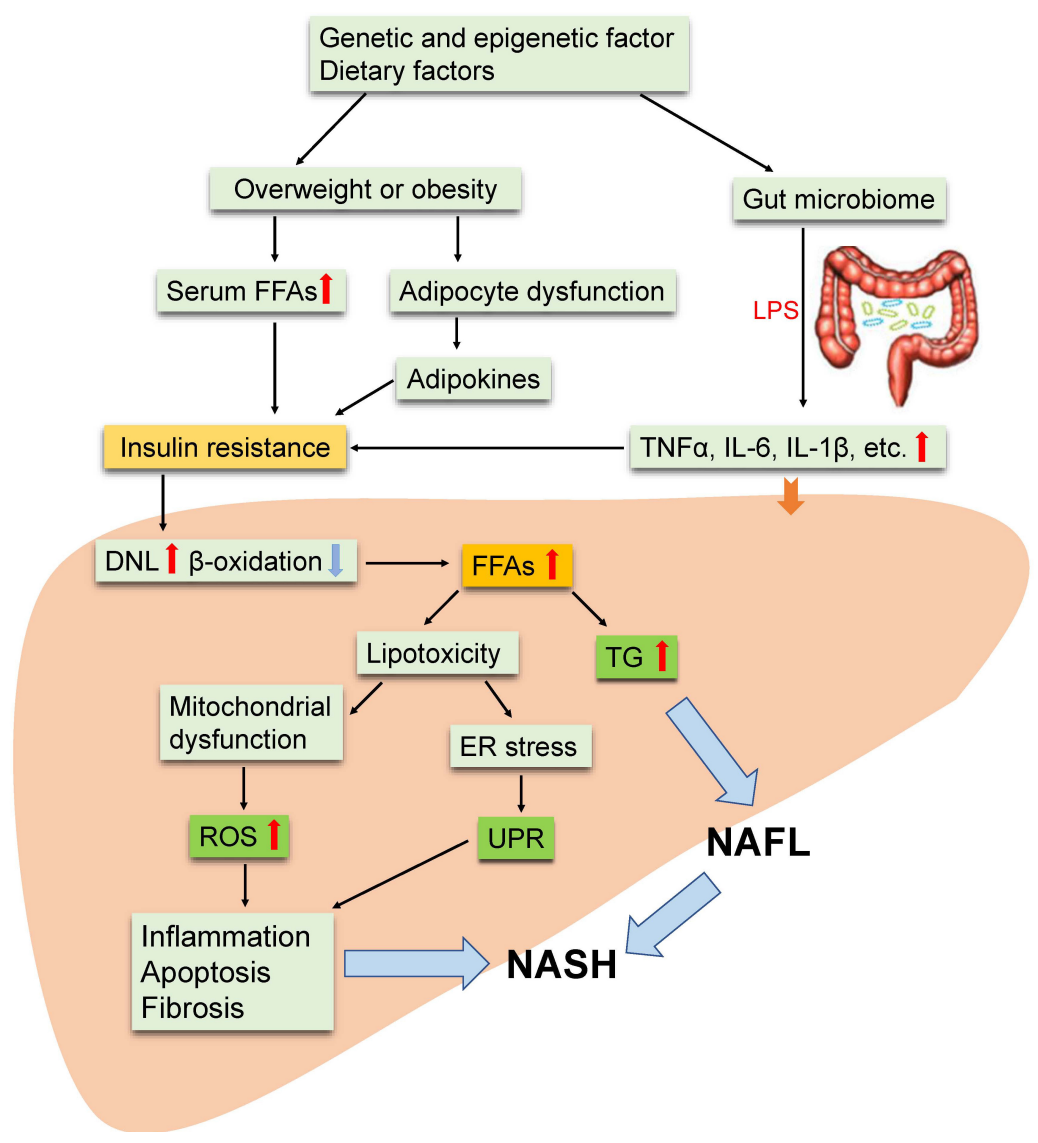

Figure 1. Multiple-hit hypothesis of the progression of NAFLD/NASH. Dietary habits, environmental, and genetic factors cause overweight or obesity and change the intestinal microbiome. This results in increased serum FFA and inflammatory factor (adipo-, cytoand/or chemokines) levels and eventually leads to insulin resistance. Furthermore, insulin resistance leads to an increase in DNL in the liver and increases the synthesis and accumulation of TG and toxic levels of fatty acids. Fat accumulation in the liver in the form of TG leads to liver steatosis (NAFL). Free cholesterol and other lipid metabolites cause mitochondrial dysfunction and subsequent oxidative stress, and ROS release and ER stress further activates UPR; which collectively lead to hepatic inflammation and fibrosis (NASH). NAFLD: Nonalcoholic fatty liver disease; NASH: nonalcoholic steatohepatitis; FFAs: free fatty acids; DNL: de novo lipogenesis; LPS: lipopolysaccharide; TNF $\alpha$ : tumor necrosis factor alpha: IL-6: interleukin-6; TG: triglycerides; ER: endoplasmic reticulum; UPR: unfolded protein response; ROS: reactive oxygen species.

protecting the nervous system, preventing diabetes and cardiovascular diseases ${ }^{[17,24-27]}$, and it is widely used in various industries such as food, cosmetics, health care products, and aquaculture. Considering the biological characteristics of astaxanthin and the complex pathogenesis of NASH, it remains unknown whether astaxanthin can be used to treat NASH; the underlying mechanism of action also remains obscured.

\section{THE MECHANISM OF ASTAXANTHIN IN THE PREVENTION OF NASH}

\section{Anti-oxidative stress effect}

As stated above, oxidative stress is one of the various factors that contribute to the "multiple hits" in the pathogenesis of NASH and is the main contributor of liver injury and disease progression in NAFLD. Indeed, several oxidative stress biomarkers that have been determined in clinical models of NAFLD include nitric oxide (NO), lipid peroxidation products [lipid peroxides, thiobarbituric acid reactive substances (TBARS), Hydroperoxides, 8-isoprostane and 4-Hydroxynonenal], protein oxidation products (protein carbonyl, nitrotyrosine), DNA oxidation product (8-OH-dG), and CYP2E1 ${ }^{[28,29]}$. Additionally, two clinical 
studies have found that increases in oxidative stress in vivo, measured by urinary 8-iso-prostaglandin F $2 \alpha$ (8-iso-PGF2 $\alpha$ ), which is derived from the non-enzymatic oxidation of arachidonic acid and serum levels of soluble NOX2-derived peptide (sNOX2-dp), is an indicator of NOX2 activation, a NADPH oxidase isoform involved in ROS generation ${ }^{[30,31]}$.

Oxidative stress damage is due to the imbalance of oxidation and anti-oxidation processes in the body that cause tissue injury induced by excessive production of free radicals, ROS and reactive nitrogen species (RNS). Excessive ROS can react with proteins, lipids and DNA through a chain reaction, thereby destroying homeostasis and causing tissue damage ${ }^{[32,33]}$. However, studies have shown that ROS can be eliminated from their oxidative activity by antioxidants such as carotenoids. Carotenoids contain polyene chains and longchain conjugated double bonds, which are responsible for antioxidant activities, acting by quenching singlet oxygen to terminate the free radical chain reaction in the organism ${ }^{[34,35]}$. Astaxanthin, one of the most prominent carotenoids with antioxidant activity, can penetrate the whole cell membrane, reduce membrane permeability, and limit the entry of peroxide promoters such as hydrogen peroxide and tert-butyl hydroperoxide into the cell ${ }^{[36,37]}$. Thus, oxidative damage to pivotal molecules in cells can be prevented. Astaxanthin scavenges oxygen free radicals and prevents lipid auto-oxidation with a capacity that is 6,000 times greater than that of vitamin C, 800 times that of coenzyme Q10, 550 times that of vitamin E, 200 times that of tea polyphenols, and 10 times that of beta carotene ${ }^{[17,38]}$; rightfully therefore known as a "super antioxidant". Jorgensen et al. ${ }^{[39]}$ found that astaxanthin is more effective than beta-carotene and zeaxanthin in preventing excessive oxidation of unsaturated fatty acid methyl esters. This conclusion has also been verified in various biofilm models, including phosphatidylcholine liposomes ${ }^{[40]}$ and rat liver microsomes ${ }^{[37]}$.

Nakagawa et al. ${ }^{[41]}$ reported that supplementation with astaxanthin $(6$ and $12 \mathrm{mg} / \mathrm{d})$ in 30 healthy subjects decreased erythrocyte phospholipid hydroperoxide (PLOOH) levels and increased astaxanthin levels, 12 weeks after administration. An animal study showed that the concentration of catalase (CAT), superoxide dismutase (SOD) and peroxidase (POD) increased significantly in the plasma and hepatocytes of rats that were fed Haematococcus pluvialis ${ }^{[42]}$. In obese and overweight adults, astaxanthin supplementation (5 and $20 \mathrm{mg} / \mathrm{d}$ ) dramatically reduced the level of biomarkers related to oxidative stress, including malondialdehyde (MDA) and isoprostane, and increased SOD and total antioxidant capacity (TAC). These findings indicate the strong antioxidant capacity of astaxanthin in $v i v{ }^{[43]}$.

\section{Anti-inflammatory effect and enhancement in M2 macrophage polarization}

Given the strong link between inflammation and oxidative stress, it is not surprising that astaxanthin has been studied as an agent to attenuate inflammation. In vitro, astaxanthin has been shown to reduce proinflammatory markers in several cell lines, such as rat alveolar macrophages ${ }^{[44]}$, U937 cells ${ }^{[45]}$, RAW 264.7 cells $^{[46]}$, Thp-1 cells ${ }^{[24]}$, proximal renal tubular epithelial cells ${ }^{[47]}$, HUVECs ${ }^{[48]}$, and human lymphocytes ${ }^{[4]}$.

Wan et al. ${ }^{[50]}$ demonstrated that M2 macrophage/Kupffer cells promote apoptosis in M1 macrophage/Kupffer cells and inhibit NAFLD progression. Astaxanthin restrains M1 macrophage/Kupffer cells and increases M2 macrophage/Kupffer cells, reducing liver recruitment of $\mathrm{CD} 4^{+}$and $\mathrm{CD} 8^{+}$cells and inhibiting inflammatory responses in $\mathrm{NAFLD}^{[51]}$. Inflammatory factors aggravate the progression of NAFLD; however, astaxanthin reduces the levels of interleukin (IL)- 6 and tumor necrosis factor (TNF) $\alpha$ in the liver through proliferator-activated receptor $\alpha$ (PPAR $\alpha)$ to alleviate inflammation ${ }^{[2]}$. When NASH was induced by diet, administration of natural astaxanthin $(0.02 \%, \approx 20 \mathrm{mg} / \mathrm{kg} \mathrm{BW})$ reduced liver inflammation and insulin resistance in $\mathrm{C} 57 \mathrm{BL} / 6 \mathrm{~J}$ mice ${ }^{[51]}$. Compared to vitamin E, astaxanthin was more effective in preventing and treating NASH in this animal model ${ }^{[51]}$. 
Recently, the gut-liver axis has been shown to mediate the NASH progression ${ }^{[53]}$. Notably, liver lipopolysaccharide (LPS) is produced by intestinal microbiota, which were known to induce oxidative stress and inflammation. The LPS/toll-like receptor 4 (TLR4)/nuclear factor-kappa B (NF- $\mathrm{BB}$ ) signaling is critical for the activation of inflammatory pathways associated with $\mathrm{NASH}^{[54]}$. Lumeng et al..$^{[55]}$ found that in primary macrophages and RAW 264.7 cells stimulated by LPS, astaxanthin significantly reduced the levels of NO, prostaglandin E2 (PGE2), TNF $\alpha$, and IL-1 $\beta$ by inhibiting NF- $\kappa$ B activation. Macedo et al. ${ }^{[56]}$ showed that astaxanthin can obviously reduce the production of proinflammatory cytokines, such as TNF $\alpha$ and IL6 , secreted by LPS-induced neutrophils and enhance the phagocytosis and bactericidal ability of neutrophils by inhibiting the production of $\mathrm{O}_{2}^{-}$(superoxide anion radical) and $\mathrm{H}_{2} \mathrm{O}_{2}$.

\section{Improvement of mitochondrial respiratory chain}

Numerous studies have shown that excessive ROS can cause oxidative damage to mitochondria. In turn, damage to the mitochondrial respiratory chain complex I and III releases electrons to produce a large amount of ROS; approximately $90 \%$ of ROS in cells are produced from mitochondria. Superfluous ROS cause mitochondrial structural abnormalities and functional deficits, mainly manifested in decreased mitochondrial membrane potential, mitochondrial mutation, uncoupling of the mitochondrial respiratory chain, increased free radical production, and decreased adenosine triphosphate (ATP) production. Dysfunctional mitochondria trigger inflammatory cytokine production and often lead to the development of degenerative diseases, aging, metabolic diseases, cardiovascular diseases and so on.

A growing body of studies has also involved astaxanthin in improving cellular mitochondrial oxidative respiratory chains to resist oxidative stress damage, and recent results hypothesize that astaxanthin has a "mitochondrial targeting" effect in cells. Fan et al. ${ }^{[5]}$ verified that astaxanthin significantly attenuated homocysteine-induced cytotoxicity of $\mathrm{H}_{9} \mathrm{C} 2$ cells by inhibiting mitochondria-mediated apoptosis and blocked homocysteine-induced mitochondrial dysfunction by modulating the expression of the Bcl-2 family. Importantly, astaxanthin also significantly inhibits homocysteine-induced cardiotoxicity in vivo as well as improves angiogenesis. A similar study has shown that astaxanthin has the potential to reverse homocysteine-induced neurotoxicity and apoptosis by inhibiting mitochondrial dysfunction and ROSmediated oxidative damage and regulating the MAPK and AKT pathways ${ }^{[58]}$. After treatment of gastric epithelial cells with astaxanthin, Kim et al. ${ }^{[59]}$ found that Helicobacter pylori-induced increases in ROS, mitochondrial dysfunction, NF- $\mathrm{KB}$ activation, and IL-8 expression were alleviated without affecting $\mathrm{NADPH}$ oxidase activity, indicating thereby that astaxanthin could prevent oxidative stress-mediated Helicobacter pylori infection that is associated with gastric inflammation $\mathrm{Yu}$ et al. ${ }^{[60]}$ also found that astaxanthin can improve heat-induced skeletal muscle oxidative damage. In mouse $\mathrm{C}_{2} \mathrm{C} 12$ myoblasts exposed to heat stress at $43{ }^{\circ} \mathrm{C}$, astaxanthin lessened heat-induced ROS production in a concentrationdependent manner (1-20 $\mu \mathrm{M})$, preventing mitochondrial disruption, depolarization and apoptotic cell death. Astaxanthin increases the protein expression of peroxisome proliferator-activated receptor $\gamma$ coactivator-1 $\beta$ (PGC-1 $\alpha$ ) and mitochondrial transcription factor A (TFAM) at $37{ }^{\circ} \mathrm{C}$, and maintains mitochondrial tubular structure and a normal membrane potential $(\Delta \Psi \mathrm{m})$, i.e., maintainance of mitochondrial integrity and function. Data from Mano et al. ${ }^{[61]}$ showed that astaxanthin inhibits oxidation and nitridation, which leads to apoptosis and lipid peroxidation of cytochrome $\mathrm{c}$ peroxidase, although its efficiency varies with membrane $\mathrm{pH}$ and lipid composition. It is hypothesized that astaxanthin is endowed with $\mathrm{pH}$-dependent antioxidant/antiapoptotic properties in respiratory mitochondria. Astaxanthin and tocopherol nanoemulsions (NEs) were prepared using sodium caseinate (AS-AT/SC NEs) as a raw material to protect cells from ROS, oxidative stress, and mitochondrial membrane potential through mitochondriamediated apoptosis to prevent cell death, demonstrating therefore that apoptosis induced by AS-AT/SC NEs may be a potential method to destroy cancer cells ${ }^{[62]}$. Taken together, astaxanthin can ameliorate mitochondrial glutathione (GSH) activity, complex I activity, ATPase activity, mitochondrial membrane 
potential and fluidity by ROS, and excessively open the membrane permeability transition pore (MPTP) ${ }^{[63]}$.

Although an accurate discussion is lacking on the mechanisms by which mitochondria might assist in the prevention and treatment of NASH, the above studies suggest that mitochondrial function plays an indelible role in NASH.

\section{Lipid metabolism}

Jia et al..$^{[52]}$ administered astaxanthin orally to C57BL/6J mice that were fed a high-fat diet for 8 weeks and found that astaxanthin could improve liver lipid accumulation and decrease liver TG levels. Astaxanthin was found to regulate PPAR levels. Activated PPAR $\alpha$ increases liver fatty acid transport, metabolism, and oxidation levels; inhibits liver fat accumulation; induces hepatocyte autophagy; and cleaves lipid droplets through AMP-activated protein kinase/PGC-1 $\alpha^{[64-66]}$. PPAR $\alpha$ has become a key target for the treatment of NAFLD - activation of PPAR $\gamma$ regulates lipid synthesis-related gene expression and promotes fatty acid storage, and PPAR $\gamma$ overexpression induces hepatic lipid accumulation ${ }^{[67]}$. Astaxanthin activates PPAR $\alpha$, inhibits PPAR $\gamma$ expression, and reduces intrahepatic fat synthesis ${ }^{[63]}$. In addition, astaxanthin inhibits the AKT-mTOR pathway, which also causes autophagy of liver cells and breaks down lipid droplets stored in the liver ${ }^{[52]}$. Furthermore, astaxanthin reduces liver fatty acid synthesizing enzyme (FASN) mRNA levels and directly inhibits de novo synthesis of fat ${ }^{[68]}$.

In vitro and clinical studies have shown that the continuous use of astaxanthin for 2 weeks can significantly prolong the oxidation time of low-density lipoprotein (LDL). Astaxanthin can inhibit the production of oxLDL and the utilization of ox-LDL by the activation of macrophages along with the enhanced expression of PPAR $\alpha$. At the same time, it inhibits the expression of PPAR $\gamma$ and reduces the synthesis of fat in the liver. Augusti et al. ${ }^{[69]}$ found that astaxanthin can significantly reduce oxidative stress damage and program lower blood lipid levels in rabbits with hypercholesterolemia. In patients with hypertriglyceridemia, serum TG levels of patients with long-term oral astaxanthin were significantly lower than those in the control group, while adiponectin and high-density lipoprotein (HDL) levels were significantly increased ${ }^{[70]}$. In obese and overweight people, oral astaxanthin lowers apolipoprotein B and LDL cholesterol levels and improves oxidative damage $\mathrm{e}^{[43]}$. It has been reported that astaxanthin also reduces platelet aggregation and promote fibrinolytic activity in rats with hyperlipidemia induced by a high-fat diet. These positive effects are related to decreased serum lipid and lipoprotein levels, antioxidant production, and protection of endothelial cells ${ }^{[71]}$.

\section{Amelioration of insulin resistance}

Insulin resistance is the major contributor to obesity, type 2 diabetes, and NAFLD. Insulin activates the tyrosine kinase activity of insulin receptor-beta (IR $\beta$ ) subunits, which phosphorylates insulin receptor substrate, IRS, to activate the insulin metabolism pathway: IRS1-PI3K-AKT (insulin receptor substrate 1, phosphoinositide 3-kinase, serine/threonine kinase). When serine/threonine protein kinases, such as Jun Nterminal kinases (JNK) and mitogen-activated protein kinase 3 (ERK-1), inhibitors of nuclear factor kappaB kinase $(\mathrm{NF}-\kappa \mathrm{B})$ subunit beta (IKK- $\beta$ ), phosphorylate IR $\beta$ and IRS1, the IRS1-PI3K-AKT pathway is blocked and its activity decreases, and insulin resistance occurs accordingly ${ }^{[2,73]}$. The activation of JNK and NF- $\mathrm{KB}$, stimulated by sustained endoplasmic reticulum (ER) stress also causes insulin resistance ${ }^{[74]}$. Bhuvaneswari et al. ${ }^{[75,76]}$ established a mouse model of type 2 diabetes induced by a high fructose-fat diet (HFFD) to study the effects of astaxanthin on hepatic insulin signaling and glucose metabolism. Astaxanthin intervention significantly improved insulin sensitivity in the HFFD group. This is because astaxanthin restores the insulin metabolic pathway by decreasing the activity of JNK and ERK-1 to ameliorate insulin resistance in the liver. Ni et al. ${ }^{[51]}$ constructed a diet-induced NASH mouse model that consisted of a high-fat, high cholesterol, and cholate diet (CL). In the fasting and fed states, glucose 
intolerance and hyperinsulinemia that accompanied the CL diet were significantly ameliorated by astaxanthin, and vitamin $\mathrm{E}$ treatment also reduced the plasma insulin levels to some extent. This result was related to the fact that astaxanthin-treated mice produced more insulin-stimulated phosphorylated IR $\beta$ and AKT than did control mice, while vitamin E had little effect on hepatic insulin signaling.

\section{Anti-fibrosis effect}

Hepatic fibrosis is a wound healing response characterized by excessive accumulation of extracellular matrix. Hepatic stellate cell (HSC) activation plays a key role in liver fibrosis ${ }^{[77]}$. Transforming growth factor beta 1 (TGF- $\beta 1)$ is the most effective profibrotic cytokine ${ }^{[78]}$. TGF- $\beta 1 / \mathrm{Smad} 3$ signaling regulates transcription of key genes in fibrosis, causing liver fibrosis. The imbalance in matrix metalloproteinases (MMPs) and MMP inhibitors (tissue inhibitor of MMPs, TIMPs) accelerates the progression of liver fibrosis, and TGF- $\beta 1$ regulates the expression of MMPs and TIMPs. Yang et al. ${ }^{[79]}$ confirmed that astaxanthin can reduce the expression of the TGF- $\beta 1$-induced fibrosis genes $\alpha$-SMA and Col 1 A 1 and effectively inhibit fibrosis in LX-2 cells. The mechanism is that astaxanthin downregulates Smad3 phosphorylation and nuclear translocation induced by TGF- $\beta 1$. Yang et al. ${ }^{[79]}$ also observed that when activated primary HSCs were coincubated with astaxanthin, $\alpha$-SMA mRNA and protein levels were significantly downregulated, indicating that astaxanthin can inhibit the early activation of resting HSCs. Shen et al. ${ }^{[80]}$ studied the protective effect of astaxanthin on liver fibrosis induced by carbon tetrachloride $(\mathrm{CCl} 4)$ and bile duct ligation. The results showed that astaxanthin effectively improved the pathological damage of liver fibrosis. Astaxanthin reduces the expression of TGF- $\beta 1$ by downregulating nuclear NF- $\mathrm{kB}$ levels, maintains the balance between MMP2 and TIMP1, and inhibits the activation of HSCs and the formation of extracellular matrix (ECM). Hernandez-Gea et al ${ }^{[81]}$ reported that autophagic degradation of lipid droplets in HSCs provides energy for HSC activation, thereby aggravating the process of liver fibrosis. Astaxanthin alleviates HSC autophagy levels, reduces nutrient supply, and inhibits HSC activation. In addition, histone acetylation, as an epigenetic model, is involved in the activation of HSCs and the process of liver fibrosis ${ }^{[82]}$. Some studies have found that astaxanthin significantly suppresses the activation of HSCs by decreasing the expression of histone deacetylase 9 (HDAC9); HDAC3 and HDAC4 may also be involved in this process $^{[74]}$.

\section{Antitumor formation}

Hepatocellular carcinoma (HCC) is a malignant tumor with extremely high morbidity and mortality. Multiple signaling pathways, such as NF-кB p65, Wnt/beta-catenin, JAK/STAT, Hedgehog, Ras/MAPK and so on, are closely related to the development of liver cancer ${ }^{[83,84]}$. In the aflatoxin (AFB1)-induced liver cancer model, astaxanthin markedly reduced the number and size of liver cancer lesions. In-depth studies have found that astaxanthin significantly inhibits AFB1-induced single-strand DNA breaks and AFB1 binding to liver DNA and plasma albumin ${ }^{[85]}$. Tripathi et al. ${ }^{[86]}$ found that astaxanthin inhibits cyclophosphamide-induced liver tumors in the early stage in rats. The Nrf2-ARE pathway is an endogenous antioxidant stress pathway that regulates the expression of SOD and DNA repair enzymes (e.g., OGG1, XRCC1, XPD, and XPG), which play an important role in tumorigenesis and progression ${ }^{[8,88]}$. Astaxanthin can inhibit the occurrence and development of liver cancer, which in turn, may be related to the activation of Nrf2-ARE pathway.

Song et al ${ }^{[89]}$ confirmed that astaxanthin induces mitochondria-mediated apoptosis in the mouse liver cancer cell line CBRH-7919 by inhibiting the JAK/STAT3 pathway. In addition, astaxanthin inhibits liver tumorigenesis and may be involved in the regulation of NM23, which encodes a nucleoside diphosphate kinase. The upregulated expression of this protein is beneficial to the correct assembly of the cytoskeleton and the transmission of $\mathrm{T}$ protein signaling. Li et al..$^{\left[{ }^{\circ 0]}\right.}$ confirmed that astaxanthin can prevent the proliferation of human hepatoma cells LM3 and SMMC-7721 and induce their apoptosis. The possible 


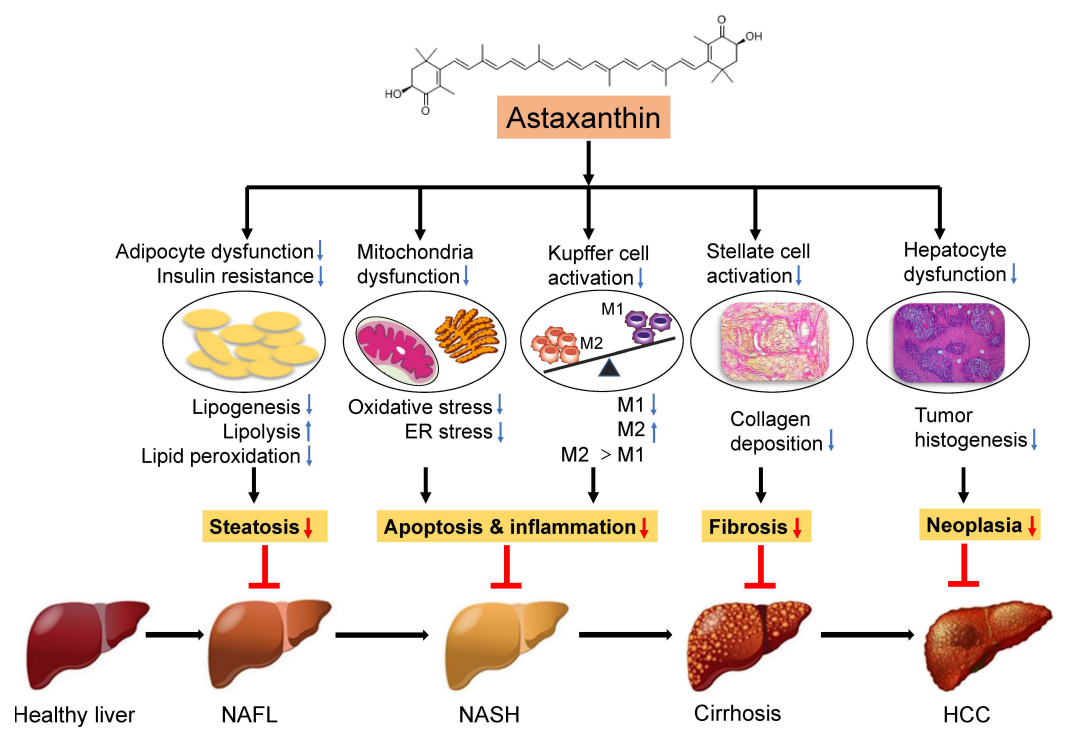

Figure 2. Mechanisms of the effects of astaxanthin on NASH. The strong antioxidant effect of astaxanthin can significantly inhibit oxidative stress, thereby reducing mitochondrial damage and endoplasmic reticulum stress, leading to a shift to M2 macrophage polarization and ultimately reversing liver steatosis, inflammation, and insulin resistance. Moreover, astaxanthin can reduce the activation of hepatic stellate cells to ameliorate hepatic fibrosis as a result of M1/M2 macrophage transformation. In addition, astaxanthin can inhibit the generation of hepatocyte tumors. Thus, astaxanthin prevents the development of NAFLD by inhibiting lipid accumulation, inflammation, and fibrosis in the liver. NAFLD: Nonalcoholic fatty liver disease; NASH: nonalcoholic steatohepatitis; KC: Kupffer cell; M1: proinflammatory macrophages; M2: anti-inflammatory macrophages; HSC: hepatic stellate cells; HCC: hepatocellular carcinoma.

mechanism is that astaxanthin inhibits the NF- $\mathrm{kB}$ and Wnt/beta-catenin signaling pathways and regulates downstream the expression of the antiapoptotic protein, Bcl-2, and apoptosis-related gene, Bax; thereby promoting apoptosis of tumor cells. At the same time, astaxanthin decreases the phosphorylation of glycogen synthetase kinase-3 beta (GSK-3 $\beta$ ) and inhibits tumor cell infiltration. Consistent with these findings, Shao et al. ${ }^{[91]}$ showed that astaxanthin can inhibit the proliferation of mouse hepatoma cell line $\mathrm{H} 22$ and arrest cells in the mitotic G2 phase.

The increase in de novo fat synthesis is a common feature of many malignant tumors. FASN is expressed in a variety of malignant tumors, including liver cancer ${ }^{[92,93]}$, and astaxanthin downregulates liver FASN mRNA levels to restrain liver tumorigenesis ${ }^{[94]}$. In addition, adipocytokines, another key regulator of the fat synthesis pathway, play an important role in repressing the proliferation of human liver tumor cells and inducing apoptosis. Astaxanthin observably inhibits liver tumorigenesis in obese mice by increasing serum adipocytokine levels ${ }^{[95]}$.

\section{CONCLUSION}

Astaxanthin has been recognized as a new food resource and is expected to have good application prospects in health food and dietary supplements. The prevalence of NASH continues to persist globally, and its complex and diverse pathogenesis makes it difficult to diagnose and treat. Currently, there are no FDAapproved standard drug regimens in the guidelines for management of NASH. At present, great progress has been made in researching the antioxidant activity of astaxanthin. A large number of studies have demonstrated that astaxanthin can prevent or treat NASH through various mechanisms [Figure 2]. Most studies on the influence of astaxanthin on NASH remain at the cellular and animal experiment level. However, the presented research in this work on the prevention and treatment of NASH by astaxanthin indicates that there is broad potential and hope for the application of astaxanthin in NASH. As a futuristic 
goal, we still need large-scale clinical trials to verify the actual effects of astaxanthin on the human body and provide a theoretical basis to continually explore the beneficial effects of astaxanthin antioxidant activity on the human body.

\section{DECLARATIONS}

\section{Authors' contributions}

Contributed to the drafting and writing of the manuscript: Gao LJ, Zhu YQ, Xu L

\section{Availability of data and materials}

Not applicable.

\section{Financial support and sponsorship}

This study was supported by Xinmiao Talents Program of Zhejiang Province granted to L.G (2019R413070) and Wenzhou Municipal Science and Technology Bureau granted to L.X. (Y20190049).

\section{Conflicts of interest}

All authors declared that there are no conflicts of interest.

\section{Ethical approval and consent to participate}

Not applicable.

\section{Consent for publication}

Not applicable.

\section{Copyright}

(c) The Author(s) 2021.

\section{REFERENCES}

1. Rinella ME, Tacke F, Sanyal AJ, Anstee QM, Abdelmalek MF; participants of the AASLD/EASL Workshop. Report on the AASLD/EASL joint workshop on clinical trial endpoints in NAFLD. J Hepatol 2019;71:823-33. DOI PubMed

2. Caldwell S, Argo C. The natural history of non-alcoholic fatty liver disease. Dig Dis 2010;28:162-8. DOI PubMed

3. Friedman SL, Neuschwander-Tetri BA, Rinella M, Sanyal AJ. Mechanisms of NAFLD development and therapeutic strategies. Nat Med 2018;24:908-22. DOI PubMed PMC

4. Kulik L, El-Serag HB. Epidemiology and management of hepatocellular carcinoma. Gastroenterology 2019;156:477-91.e1. DOI PubMed PMC

5. Younossi ZM, Koenig AB, Abdelatif D, Fazel Y, Henry L, Wymer M. Global epidemiology of nonalcoholic fatty liver disease-metaanalytic assessment of prevalence, incidence, and outcomes. Hepatology 2016;64:73-84. DOI PubMed

6. Zhou JH, Zhou F, Wang WX, et al. Epidemiological features of NAFLD from 1999 to 2018 in China. Hepatology 2020;71:1851-64. DOI PubMed

7. Zhu JZ, Dai YN, Wang YM, Zhou QY, Yu CH, Li YM. Prevalence of nonalcoholic fatty liver disease and economy. Dig Dis Sci 2015;60:3194-202. DOI PubMed

8. Wong RJ, Aguilar M, Cheung R, et al. Nonalcoholic steatohepatitis is the second leading etiology of liver disease among adults awaiting liver transplantation in the United States. Gastroenterology 2015;148:547-55. DOI PubMed

9. Buzzetti E, Pinzani M, Tsochatzis EA. The multiple-hit pathogenesis of non-alcoholic fatty liver disease (NAFLD). Metabolism 2016;65:1038-48. DOI PubMed

10. Basaranoglu M. From fatty liver to fibrosis: A tale of "second hit". World J of Gastroenterol 2013;19:1158. DOI PubMed PMC

11. Ni Y, Zhuge F, Nagashimada M, et al. Lycopene prevents the progression of lipotoxicity-induced nonalcoholic steatohepatitis by decreasing oxidative stress in mice. Free Radical Bio Med 2020;152:571-82. DOI PubMed

12. Schuppan D, Schattenberg JM. Non-alcoholic steatohepatitis: pathogenesis and novel therapeutic approaches. J Gastroenterol Hepatol 2013;28 Suppl 1:68-76. DOI PubMed

13. Farrell GC, Larter CZ. Nonalcoholic fatty liver disease: From steatosis to cirrhosis. Hepatology 2006;43:S99-S112. DOI PubMed

14. Lavine JE. Effect of vitamin E or metformin for treatment of nonalcoholic fatty liver disease in children and adolescents. JAMA 2011;305:1659. DOI

15. Bril F, Biernacki DM, Kalavalapalli S, et al. Role of vitamin E for nonalcoholic steatohepatitis in patients with type 2 diabetes: A 
randomized controlled trial. Diabetes Care 2019;42:1481-8. DOI PubMed

16. Sanyal AJ, Chalasani N, Kowdley KV, et al. Pioglitazone, vitamin E, or placebo for nonalcoholic steatohepatitis. New Engl J Med 2010;362:1675-85. DOI PubMed PMC

17. Ambati RR, Phang SM, Ravi S, Aswathanarayana RG. Astaxanthin: sources, extraction, stability, biological activities and its commercial applications-a review. Mar Drugs 2014;12:128-52. DOI PubMed PMC

18. Krinsky NI. Antioxidant functions of carotenoids. Free Radic Biol Med 1989;7:617-35. DOI PubMed

19. Rishton GM. Natural products as a robust source of new drugs and drug leads: past successes and present day issues. Am J Cardiol 2008;101:43d-9d. DOI PubMed

20. Guerin M, Huntley ME, Olaizola M. Haematococcus astaxanthin: applications for human health and nutrition. Trends Biotechnol 2003;21:210-6. DOI PubMed

21. Spiller GA, Dewell A. Safety of an astaxanthin-rich Haematococcus pluvialis algal extract: a randomized clinical trial. J Med Food 2003;6:51-6. DOI PubMed

22. Fassett RG, Coombes JS. Astaxanthin in cardiovascular health and disease. Molecules 2012;17:2030-48. DOI PubMed PMC

23. Hussein G, Sankawa U, Goto H, Matsumoto K, Watanabe H. Astaxanthin, a carotenoid with potential in human health and nutrition. $J$ Nat Prod 2006;69:443-9. DOI PubMed

24. Kishimoto Y, Tani M, Uto-Kondo H, et al. Astaxanthin suppresses scavenger receptor expression and matrix metalloproteinase activity in macrophages. Eur J Nutr 2010;49:119-26. DOI PubMed

25. Lobos P, Bruna B, Cordova A, et al. Astaxanthin protects primary hippocampal neurons against noxious effects of a beta-oligomers. Neural Plast 2016;2016:3456783. DOI PubMed PMC

26. Sila A, Ghlissi Z, Kamoun Z, et al. Astaxanthin from shrimp by-products ameliorates nephropathy in diabetic rats. Eur $J$ Nutr 2015;54:301-7. DOI PubMed

27. Visioli F, Artaria C. Astaxanthin in cardiovascular health and disease: mechanisms of action, therapeutic merits, and knowledge gaps. Food \& Function 2017;8:39-63. DOI PubMed

28. Sutti S, Jindal A, Locatelli I, et al. Adaptive immune responses triggered by oxidative stress contribute to hepatic inflammation in NASH. Hepatology 2014;59:886-97. DOI PubMed

29. Polimeni L, Del Ben M, Baratta F, et al. Oxidative stress: new insights on the association of non-alcoholic fatty liver disease and atherosclerosis. World J Hepatol 2015;7:1325-36. DOI PubMed PMC

30. Del Ben M, Polimeni L, Baratta F, et al. Serum cytokeratin-18 is associated with $\mathrm{NOX}_{2}$-Generated oxidative stress in patients with nonalcoholic fatty liver. Int J Hepatol 2014;2014:784985. DOI PubMed PMC

31. Del Ben M, Polimeni L, Carnevale R et al. $\mathrm{NOX}_{2}$-generated oxidative stress is associated with severity of ultrasound liver steatosis in patients with non-alcoholic fatty liver disease. BMC Gastroenterol 2014;14:1-8. DOI PubMed PMC

32. Betteridge DJ. What is oxidative stress? Metabolism 2000;49:3-8. DOI PubMed

33. Touyz RM. Reactive oxygen species, vascular oxidative stress, and redox signaling in hypertension - What is the clinical significance? Hypertension 2004;44:248-52. DOI PubMed

34. Chen G, Ni Y, Nagata N, Xu L, Ota T. Micronutrient antioxidants and nonalcoholic fatty liver disease. Int J Mol Sci 2016;17:1379. DOI PubMed PMC

35. Meinke MC, Friedrich A, Tscherch K, et al. Influence of dietary carotenoids on radical scavenging capacity of the skin and skin lipids. Eur J Pharm Biopharm 2013;84:365-73. DOI PubMed

36. Barros MP, Pinto E, Colepicolo P, Pedersen M. Astaxanthin and peridinin inhibit oxidative damage in Fe(2+)-loaded liposomes: scavenging oxyradicals or changing membrane permeability? Biochem Biophys Res Commun 2001;288:225-32. DOI PubMed

37. Palozza P, Krinsky NI. Astaxanthin and canthaxanthin are potent antioxidants in a membrane model. Arch Biochem Biophys 1992;297:291-5. DOI PubMed

38. Zhang ZW, Xu XC, Liu T, Yuan S. Mitochondrion-permeable antioxidants to treat ROS-burst-mediated acute diseases. Oxid Med Cell Longev 2016;2016:6859523. DOI PubMed PMC

39. Jorgensen K, Skibsted LH. Carotenoid scavenging of radicals. Effect of carotenoid structure and oxygen partial pressure on antioxidative activity. Z Lebensm Unters Forsch 1993;196:423-9. DOI PubMed

40. Lim BP, Nagao A, Terao J, Tanaka K, Suzuki T, Takama K. Antioxidant activity of xanthophylls on peroxyl radical-mediated phospholipid peroxidation. Biochim Biophys Acta 1992;1126:178-84. DOI PubMed

41. Nakagawa K, Kiko T, Miyazawa T, et al. Antioxidant effect of astaxanthin on phospholipid peroxidation in human erythrocytes. $\mathrm{Br} J$ Nutr 2011;105:1563-71. DOI PubMed

42. Rao A, Raghunath Reddy RL, Baskaran V, Sarada R, Ravishankar GA. Characterization of microalgal carotenoids by mass spectrometry and their bioavailability and antioxidant properties elucidated in rat model. J Agric Food Chem 2010;58:8553-9. DOI PubMed

43. Choi HD, Kim JH, Chang MJ, Kyu-Youn Y, Shin WG. Effects of astaxanthin on oxidative stress in overweight and obese adults. Phytother Res 2011;25:1813-8. DOI PubMed

44. Santos SD, Cahu TB, Firmino GO, et al. Shrimp waste extract and astaxanthin: rat alveolar macrophage, oxidative stress and inflammation. J Food Sci 2012;77:H141-6. DOI PubMed

45. Franceschelli S, Pesce M, Ferrone A, et al. Astaxanthin treatment confers protection against oxidative stress in U937 cells stimulated with lipopolysaccharide reducing O2- production. PLoS One 2014;9:e88359. DOI PubMed PMC

46. Lee SJ, Bai SK, Lee KS, et al. Astaxanthin inhibits nitric oxide production and inflammatory gene expression by suppressing I (kappa) B kinase-dependent NF-kappaB activation. Mol Cells 2003;16:97-105. PubMed 
47. Kim YJ, Kim YA, Yokozawa T. Protection against oxidative stress, inflammation, and apoptosis of high-glucose-exposed proximal tubular epithelial cells by astaxanthin. J Agric Food Chem 2009;57:8793-7. DOI PubMed

48. Abdelzaher LA, Imaizumi T, Suzuki T, Tomita K, Takashina M, Hattori Y. Astaxanthin alleviates oxidative stress insults-related derangements in human vascular endothelial cells exposed to glucose fluctuations. Life Sci 2016;150:24-31. DOI PubMed

49. Campoio TR, Oliveira FA, Otton R. Oxidative stress in human lymphocytes treated with fatty acid mixture: role of carotenoid astaxanthin. Toxicol In Vitro 2011;25:1448-56. DOI PubMed

50. Wan J, Benkdane M, Teixeira-Clerc F, et al. M2 Kupffer cells promote M1 Kupffer cell apoptosis: a protective mechanism against alcoholic and nonalcoholic fatty liver disease. Hepatology 2014;59:130-42. DOI PubMed

51. Ni Y, Nagashimada M, Zhuge F, et al. Astaxanthin prevents and reverses diet-induced insulin resistance and steatohepatitis in mice: A comparison with vitamin E. Sci Rep 2015;5:17192. DOI PubMed PMC

52. Jia Y, Wu C, Kim J, Kim B, Lee SJ. Astaxanthin reduces hepatic lipid accumulations in high-fat-fed C57BL/6J mice via activation of peroxisome proliferator-activated receptor (PPAR) alpha and inhibition of PPAR gamma and Akt. J Nutr Biochem 2016;28:9-18. DOI PubMed

53. Ni Y, Ni L, Zhuge F, Fu Z. The gut microbiota and its metabolites, novel targets for treating and preventing non-alcoholic fatty liver disease. Mol Nutr Food Res 2020;64:e2000375. DOI PubMed

54. Ferro D, Baratta F, Pastori D, et al. New insights into the pathogenesis of non-alcoholic fatty liver disease: gut-derived lipopolysaccharides and oxidative stress. Nutrients 2020:12. DOI PubMed PMC

55. Lumeng CN, Saltiel AR. Inflammatory links between obesity and metabolic disease. J Clin Invest 2011;121:2111-7. DOI PubMed PMC

56. Macedo RC, Bolin AP, Marin DP, Otton R. Astaxanthin addition improves human neutrophils function: in vitro study. Eur J Nutr 2010;49:447-57. DOI PubMed

57. Fan CD, Sun JY, Fu XT, et al. Astaxanthin attenuates homocysteineinduced cardiotoxicity in vitro and in vivo by inhibiting mitochondrial dysfunction and oxidative damage. Front Physiol 2017;8:1041. DOI PubMed PMC

58. Wang XJ, Chen W, Fu XT. Reversal of homocysteine-induced neurotoxicity in rat hippocampal neurons by astaxanthin: evidences for mitochondrial dysfunction and signaling crosstalk. Cell Death Discov 2018;4:50. DOI PubMed PMC

59. Kim SH, Lim JW, Kim H. Astaxanthin Inhibits Mitochondrial Dysfunction and Interleukin-8 Expression in Helicobacter pyloriInfected Gastric Epithelial Cells. Nutrients 2018:10. DOI PubMed PMC

60. Yu T, Dohl J, Chen Y, Gasier HG, Deuster PA. Astaxanthin inhibits mitochondrial dysfunction and interleukin-8 expression in helicobacter pylori-infected gastric epithelial cells. J Cell Physiol 2019;234:13292-302. DOI PubMed PMC

61. Mano CM, Guaratini T, Cardozo KHM, Colepicolo P, Bechara EJH, Barros MP. Astaxanthin Restrains Nitrative-Oxidative Peroxidation in Mitochondrial-Mimetic Liposomes: A Pre-Apoptosis model. Mar Drugs 2018;16:126. DOI PubMed PMC

62. Shanmugapriya K, Kim H, Kang HW. In vitro antitumor potential of astaxanthin nanoemulsion against cancer cells via mitochondrial mediated apoptosis. Int J Pharm 2019;560:334-46. DOI PubMed

63. Lee DH, Kim CS, Lee YJ. Astaxanthin protects against MPTP/MPP+-induced mitochondrial dysfunction and ROS production in vivo and in vitro. Food Chem Toxicol 2011;49:271-80. DOI PubMed PMC

64. Choi AM, Ryter SW, Levine B. Autophagy in human health and disease. N Engl J Med 2013;368:651-62. DOI PubMed

65. Harano Y, Yasui K, Toyama T, et al. Fenofibrate, a peroxisome proliferator-activated receptor alpha agonist, reduces hepatic steatosis and lipid peroxidation in fatty liver Shionogi mice with hereditary fatty liver. Liver Int 2006;26:613-20. DOI PubMed

66. Singh R, Kaushik S, Wang Y, et al. Autophagy regulates lipid metabolism. Nature 2009;458:1131-5. DOI PubMed PMC

67. Jones JR, Barrick C, Kim KA, et al. Deletion of PPAR gamma in adipose tissues of mice protects against high fat diet-induced obesity and insulin resistance. Proc Natl Acad Sci USA 2005;102:6207-12. DOI PubMed PMC

68. Venkatachalam MA, Griffin KA, Lan R, Geng H, Saikumar P, Bidani AK. Acute kidney injury: a springboard for progression in chronic kidney disease. Am J Physiol Renal Physiol 2010;298:F1078-94. DOI PubMed PMC

69. Augusti PR, Quatrin A, Somacal S, et al. Astaxanthin prevents changes in the activities of thioredoxin reductase and paraoxonase in hypercholesterolemic rabbits. J Clin Biochem Nutr 2012;51:42-9. DOI PubMed PMC

70. Ryu SK, King TJ, Fujioka K, Pattison J, Pashkow FJ, Tsimikas S. Effect of an oral astaxanthin prodrug (CDX-085) on lipoprotein levels and progression of atherosclerosis in LDLR(-/-) and ApoE(-/-) mice. Atherosclerosis 2012;222:99-105. DOI PubMed

71. Deng ZY, Shan WG, Wang SF, Hu MM, Chen Y. Effects of astaxanthin on blood coagulation, fibrinolysis and platelet aggregation in hyperlipidemic rats. Pharm Biol 2017;55:663-72. DOI PubMed PMC

72. Samuel VT, Shulman GI. The pathogenesis of insulin resistance: integrating signaling pathways and substrate flux. J Clin Invest 2016;126:12-22. DOI PubMed PMC

73. Ye J. Mechanisms of insulin resistance in obesity. Front Med 2013;7:14-24. DOI PubMed PMC

74. Yang Y, Bae M, Park YK, et al. Histone deacetylase 9 plays a role in the antifibrogenic effect of astaxanthin in hepatic stellate cells. $J$ Nutr Biochem 2017;40:172-7. DOI PubMed

75. Bhuvaneswari S, Anuradha CV. Astaxanthin prevents loss of insulin signaling and improves glucose metabolism in liver of insulin resistant mice. Can J Physiol Pharmacol 2012;90:1544-52. DOI PubMed

76. Bhuvaneswari S, Yogalakshmi B, Sreeja S, Anuradha CV. Astaxanthin reduces hepatic endoplasmic reticulum stress and nuclear factor-kappaB-mediated inflammation in high fructose and high fat diet-fed mice. Cell Stress Chaperones 2014;19:183-91. DOI PubMed PMC

77. Kisseleva T, Brenner DA. Role of hepatic stellate cells in fibrogenesis and the reversal of fibrosis. J Gastroenterol Hepatol 2007;22 Suppl 1:S73-8. DOI PubMed 
78. Baghy K, Iozzo RV, Kovalszky I. Decorin-TGFbeta axis in hepatic fibrosis and cirrhosis. J Histochem Cytochem 2012;60:262-8. DOI PubMed PMC

79. Yang Y, Kim B, Park YK, Koo SI, Lee JY. Astaxanthin prevents TGFbeta1-induced pro-fibrogenic gene expression by inhibiting Smad3 activation in hepatic stellate cells. Biochim Biophys Acta 2015;1850:178-85. DOI PubMed

80. Shen M, Chen K, Lu J, et al. Protective effect of astaxanthin on liver fibrosis through modulation of TGF-beta1 expression and autophagy. Mediators Inflamm 2014;2014:954502. DOI PubMed PMC

81. Hernandez-Gea V, Ghiassi-Nejad Z, Rozenfeld R, et al. Autophagy releases lipid that promotes fibrogenesis by activated hepatic stellate cells in mice and in human tissues. Gastroenterology 2012;142:938-46. DOI PubMed PMC

82. Zhao Q, Qin CY, Zhao ZH, Fan YC, Wang K. Epigenetic modifications in hepatic stellate cells contribute to liver fibrosis. Tohoku J Exp Med 2013;229:35-43. DOI PubMed

83. Dai W, Wang F, He L, et al. Genistein inhibits hepatocellular carcinoma cell migration by reversing the epithelial-mesenchymal transition: partial mediation by the transcription factor NFAT1. Mol Carcinog 2015;54:301-11. DOI PubMed

84. Villanueva A, Toffanin S, Llovet JM. Linking molecular classification of hepatocellular carcinoma and personalized medicine: preliminary steps. Curr Opin Oncol 2008;20:444-53. DOI PubMed PMC

85. Gradelet S, Le Bon AM, Berges R, Suschetet M, Astorg P. Dietary carotenoids inhibit aflatoxin B1-induced liver preneoplastic foci and DNA damage in the rat: role of the modulation of aflatoxin B1 metabolism. Carcinogenesis 1998;19:403-11. DOI PubMed

86. Tripathi DN, Jena GB. Astaxanthin intervention ameliorates cyclophosphamide-induced oxidative stress, DNA damage and early hepatocarcinogenesis in rat: role of Nrf2, p53, p38 and phase-II enzymes. Mutat Res 2010;696:69-80. DOI PubMed

87. Khor TO, Huang MT, Prawan A, et al. Increased susceptibility of Nrf2 knockout mice to colitis-associated colorectal cancer. Cancer Prev Res (Phila) 2008;1:187-91. DOI PubMed PMC

88. Lee JH, Khor TO, Shu L, et al. Dietary phytochemicals and cancer prevention: Nrf2 signaling, epigenetics, and cell death mechanisms in blocking cancer initiation and progression. Pharmacol Ther 2013;137:153-71. DOI PubMed PMC

89. Song X, Wang M, Zhang L, et al. Changes in cell ultrastructure and inhibition of JAK1/STAT3 signaling pathway in CBRH-7919 cells with astaxanthin. Toxicol Mech Methods 2012;22:679-86. DOI PubMed

90. Li J, Dai W, Xia Y, et al. Astaxanthin inhibits proliferation and induces apoptosis of human hepatocellular carcinoma cells via inhibition of NF-Kappa b P65 and Wnt/Beta-Catenin in vitro. Mar Drugs 2015;13:6064-81. DOI PubMed PMC

91. Shao Y, Ni Y, Yang J, Lin X, Li J, Zhang L. Astaxanthin inhibits proliferation and induces apoptosis and cell cycle arrest of mice H22 hepatoma cells. Med Sci Monit 2016;22:2152-60. DOI PubMed PMC

92. Menendez JA, Lupu R. Fatty acid synthase and the lipogenic phenotype in cancer pathogenesis. Nat Rev Cancer 2007;7:763-77. DOI PubMed

93. Postic C, Girard J. The role of the lipogenic pathway in the development of hepatic steatosis. Diabetes Metab 2008;34:643-8. DOI PubMed

94. Ohno T, Shimizu M, Shirakami Y, et al. Preventive effects of astaxanthin on diethylnitrosamine-induced liver tumorigenesis in C57/BL/KsJ-db/db obese mice. Hepatol Res 2016;46:E201-9. DOI PubMed

95. Saxena NK, Fu PP, Nagalingam A, et al. Adiponectin modulates C-jun N-terminal kinase and mammalian target of rapamycin and inhibits hepatocellular carcinoma. Gastroenterology 2010;139:1762-73. DOI PubMed PMC 